\title{
PERFIL EPIDEMIOLÓGICO DOS PRINCIPAIS TUMORES SÓLIDOS EM UMA UNIDADE DE ALTA COMPLEXIDADE EM ONCOLOGIA NO ESTADO DA AMAZÔNIA LEGAL
}

\author{
EPIDEMIOLOGICAL PROFILE OF THE MAIN SOLID TUMORS IN A HIGH \\ COMPLEXITY UNIT IN ONCOLOGY IN A STATE OF THE LEGAL AMAZON
}

\author{
Dielle de Carvalho Loureiro ${ }^{1}$ \\ Luciana da Silva Caetano ${ }^{1}$ \\ Roberto Marcel Soares Alves ${ }^{2}$ \\ Bráulio Érison França dos Santos ${ }^{2}$
}

\section{RESUMO}

Objetivo: Descrever o perfil epidemiológico dos tumores sólidos mais prevalentes em uma unidade de assistência de alta complexidade em oncologia em um estado da Amazônia Legal e correlacionar com os dados nacionais. Metodologia: Pesquisa descritiva, transversal e com análise quantitativa, da qual foram amostrados 1568 pacientes atendidos no período de janeiro de 2013 a dezembro de 2017, coletadas variáveis de data nascimento, raça/cor, sexo, cidade/estado, data da biópsia, data do óbito e CID-10/diagnóstico. Os dados foram analisados através do teste não paramétrico Qui-quadrado $\left(X^{2}\right)$ com $5 \%$ de probabilidade de erro e variáveis com valor de $\mathrm{P}$ estatístico menor que 0,05 foram consideradas estatisticamente significativas. Resultados: Dos principais tumores sólidos, $56 \%$ foram do sexo feminino; a faixa etária mais prevalente para ambos os sexos foi de 50 a 59 anos, de maioria proveniente da capital $(67,15 \%)$. Apenas $8,03 \%$ dos pacientes foram a óbito. $O$ câncer de colo de útero foi o mais prevalente com $19,57 \%$ e idades entre 40 a 49 anos $(24,62 \%)$ as mais frequentes para este câncer. Demonstrou-se que o perfil epidemiológico se enquadrou no aguardado para uma cidade da região Norte brasileira, caracterizada por padrões populacionais de baixa renda. Conclusão: Os dados obtidos por meio deste estudo permitiram traçar o perfil epidemiológico de pessoas com câncer atendidas em uma unidade de alta complexidade em oncologia do Amapá, evidenciando disparidades características encontradas na região Norte, em detrimento das outras regiões brasileiras.

DESCRITORES: Câncer. Oncologia. Epidemiologia.

\begin{abstract}
Objective: To describe the epidemiological profile of the most prevalent solid tumors in a high complexity care unit in oncology in a state of the Legal Amazon and to correlate it with the national data. Methods: Descriptive and cross-sectional survey with quantitative analysis, from 1568 patients which were treated from January 2013 to December 2017, collected variables of birth date, race/color, sex, city / state, date of biopsy, date of death and ICD-10/diagnosis. The data was analyzed using the non-parametric Chi-square test $\left(X^{2}\right)$ with $5 \%$ of error probability and variables with a statistical $P$ value of less than 0.05 were considered statistically significant. Results: Out of the main solid tumors, $56 \%$ were female; the most prevalent age group for both sexes was between 50 and 59 years old, with the majority coming from the capital $(67.15 \%)$. Only $8.03 \%$ of the patients died. Cervical cancer was the most prevalent with $19.57 \%$ and ages between 40 to 49 years $(24.62 \%)$ were more frequent for this cancer. It was demonstrated that the epidemiological profile was expected for a city in the North Brazilian region, characterized by low-income population patterns. Conclusion: The data obtained through this study allowed us to trace the epidemiological profile of people with cancer treated in a high complexity unit in oncology of Amapá, highlighting the characteristic disparities found in the Northern region, in detriment of other Brazilian regions.
\end{abstract}

DESCRIPTORS: Cancer. Oncology. Epidemiology.

1- Aluna de Graduação em Medicina, Universidade Federal do Amapá - UNIFAP, Macapá, Amapá, Brasil.

2- Professor Mestre em Ciências da Saúde, Colegiado de Medicina, Universidade Federal do Amapá - UNIFAP, Macapá, Amapá, Brasil. 


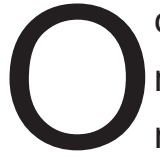
câncer é uma doença genética que reflete o desequilíbrio entre o crescimento celular e os complexos fatores que participam desse processo. Mutações nos genes responsáveis pela manutenção da proliferação celular homeostática podem iniciar e perpetuar o crescimento celular em decorrência da exposição a carcinógenos, de erros de replicação aleatória e de mecanismos de reparo de DNA defeituosos ${ }^{1-3}$.

A senescência é fator de risco importante para o desenvolvimento de neoplasias, devido às mudanças fisiológicas que aumentam a vulnerabilidade dos tecidos à carcinogênese e ao desenvolvimento de doenças crônicas, como diabetes mellitus e obesidade ${ }^{4,5}$. Além disso, cerca de $35 \%$ dos casos de câncer se devem a infecções crônicas e fatores de risco comportamentais, tais como tabagismo, alcoolismo, sedentarismo e hábitos alimentares ${ }^{6,7}$.

Em 2012, 14 milhões de novos casos e 8,2 milhões de óbitos foram documentados ao redor do mundo, com discreto predomínio do sexo masculino tanto na incidência (53\%) quanto na mortalidade (57\%) e estima-se que mais de 25 milhões de pessoas vivam com câncer diagnosticado há, pelo menos, cinco anos. Estratégias de prevenção e tratamento evoluíram nos últimos anos, porém a incidência permanece em crescimento progressivo, com estimativa de duplicação até 2030, chegando a 24 milhões de pessoas com a doença, e produção de elevados custos à população mundial ${ }^{6,8-10}$. Para tal década, também são previstos aproximadamente 13,2 milhões de óbitos por câncer no mundo, um número significativamente maior do que os 7,6 milhões ocorridos em $2008^{11}$.
O processo de transição demográfica possui forte relação com as predições de casos e óbitos por câncer para o ano de 2030. Apesar de a incidência atual ser maior nos países desenvolvidos, prevê-se que nas próximas décadas os países em desenvolvimento sejam responsáveis pela maior incidência da doença, como resultado de um contínuo desenvolvimento social e econômico, da redução das taxas de fertilidade e do crescimento populacional ${ }^{6,9,11,13}$. O mesmo é visto em relação às taxas de mortalidade que, apesar de declinantes nos países de alta renda, possuem tendência de crescimento em países de renda média e baixa, como o Brasil, sendo estes caracterizados por mortalidade precoce e possibilidade de prevenção ${ }^{9,11}$. Os idosos com mais de 65 anos, nos quais a incidência é superior em relação às outras faixas etárias, estão entre as maiores vítimas fatais da doença, representando cerca de $70 \%$ dos casos de mortes ${ }^{5}$.

Segundo o Instituto Nacional do Câncer (INCA), estima-se, para o biênio 20182019, que o Brasil tenha 600 mil novos casos de câncer em cada ano. Em nosso país, a distribuição epidemiológica indica a ocorrência de uma transição que demonstra elevação de casos de câncer mais comuns em níveis socioeconômicos mais elevados (mama, próstata, cólon e reto), com a contrapartida de que neoplasias de associação maior com níveis socioeconômicos mais baixos (colo de útero, pênis, estômago e cavidade oral) permanecem com taxas elevadas. Para 2011 a 2030, contudo, foi estimada tendência de aumento da mortalidade por câncer apenas para as regiões Norte e Nordeste, evidenciando-se decréscimo e/ou estabilidade para 
demais regiões ${ }^{11,13,14}$. Dadas as dimensões continentais do Brasil e suas grandes discrepâncias regionais e sociais, não é surpresa que haja diferenças na evolução da mortalidade por câncer. Grandes contrastes são vistos na mortalidade por câncer de colo uterino e mama devido à desigualdade entre oferta e acesso ao rastreamento, diagnóstico e tratamento. Isso aponta para a relevância de se mapear as diferenças regionais quanto a outros tipos de câncer no Brasil ${ }^{12}$.

Apesar de incidência e mortalidade serem consideradas as principais medidas estatísticas para o câncer, a prevalência é um importante indicador suplementar para o planejamento de estratégias de prestação de serviços ${ }^{15,16}$. Em 2008, a prevalência global de 5 anos para todos os tipos de câncer combinados é estimada em 28,8 milhões, com porcentagem significativamente maior em áreas com Índice de Desenvolvimento Humano (IDH) muito altas (47,2\%), quando comparadas àquelas com baixo IDH $(1,7 \%)$ e médio (35,8\%). Além disso, para o período estudado, 111 países apresentaram o câncer de próstata como o mais prevalente em homens. Já para mulheres, em 145 países, as taxas do câncer de mama corresponderam à maior porcentagem da prevalência ${ }^{15}$. Em relação à prevalência brasileira, entre as edições de 2003 e 2008 da Pesquisa Nacional de Amostra Domiciliar (PNAD) houve aumento de $33 \%$ da prevalência, sendo que na edição de 2008 a prevalência de câncer, dentre as doenças crônicas analisadas, foi de $0,56 \%{ }^{17}$.

Tanto o câncer em si quanto seu tratamento acarretam diversas alterações biológicas e sociais, causando interferências nas atividades de vida diária e nas relações no âmbito familiar. Associados ao estigma de dor e sofrimento, podem também levar os afetados a desenvolver transtornos psicológicos $^{5,8}$. A coleta de informações estatísticas desempenha um papel importante, pois tem o potencial de identificar necessidades e direcionar recursos para as diversas regiões do globo, incluindo comunidades que são mais marginalizadas, para prevenção e tratamento da doença, bem como viabilizar a criação de programas nacionais e regionais para o controle do câncer, reconhecendo-se que a prevenção e a identificação da doença em seus estágios iniciais contribuem para o sucesso do tratamento e consequente redução nas taxas de morbimortalidade $\mathrm{e}^{10,11,13}$.

Entretanto, embora a Organização Mundial de Saúde (OMS) reúna taxas globais de câncer, os dados são inconsistentes e inexistentes em muitas áreas e o processo de cura ainda constitui o centro do modelo de saúde atual, dificultando ainda mais a resolução de certos problemas, principalmente relacionados às neoplasias. No Brasil, há uma permanente dificuldade em se manter e atualizar os registros sobre a doença, devendo-se levar tal revés em consideração todas as vezes que uma avaliação do risco de câncer no país seja realizada ${ }^{10,11}$. Além disso, a associação da falta de instrução, de estruturas adequadas e de recursos financeiros refletem a elevada incidência de câncer no Brasil $^{8}$.

Portanto, os estudos epidemiológicos e programas de vigilância em saúde são fundamentais no fornecimento de conhecimentos necessários para direcionar de maneira igualitária os recursos para cada região, prin- 
cipalmente as mais carentes ${ }^{8}$. Diante disso e das grandes diferenças regionais no Brasil, o presente estudo teve como objetivo descrever o perfil epidemiológico dos principais tumores sólidos assistidos em uma unidade de assistência de alta complexidade em oncologia em um estado da Amazônia Legal e correlacionar com os dados nacionais ${ }^{12}$.

\section{METODOLOGIA}

O estudo é de caráter descritivo, transversal e com análise quantitativa. Coletaram-se 1859 dados do Registro dos Pacientes da Unidade de Assistência de Alta Complexidade em Oncologia do Amapá (UNACON-AP) obtidos através do Sistema de Gerenciamento Estadual em Saúde do Amapá (SIGES-AP), do Hospital de Clínicas Doutor Alberto Lima (HCAL), Macapá, Amapá, Brasil, dos pacientes atendidos no período de janeiro de 2013 a dezembro de 2017. A coleta realizou-se no período de junho a julho de 2018, na qual se obtiveram como variáveis a data de nascimento, raça/ cor, sexo, cidade/estado, data da biópsia, data do óbito e CID-10/diagnóstico. Realizaram-se análises retrospectivas da prevalência dos principais tumores sólidos nesta região da Amazônia, objetivando-se traçar um perfil epidemiológico desses tumores.

Nesta pesquisa, incluíram-se, pacientes portadores de tumores sólidos, assistidos na UNACON-AP, cadastrados no SIGES-AP, excluindo-se 291 clientes portadores de tumores hematológicos, tecidos moles, melanomas e não melanomas e outros diagnósticos que não de tumores sólidos, totalizando 1568 pacientes da amostragem.
Processaram-se os dados em planiIhas do Microsoft Excel 2010 e analisaram-nos através do teste não paramétrico Qui-quadrado $\left(X^{2}\right)$ com $5 \%$ de probabilidade de erro e utilizou-se o programa Bioestat 4.0 para a realização dos testes estatísticos. Variáveis com valor de $\mathrm{P}$ estatístico menor que 0,05 consideraram-se estatisticamente significativo.

A pesquisa foi inserida na Plataforma Brasil e aprovada pelo Comitê de Ética em Pesquisa (CEP) da Universidade Federal do Amapá (UNIFAP) (Parecer 2.712.734, de 14 de junho de 2018). Respeitaram-se todos os preceitos e orientações referentes à ética em pesquisa envolvendo seres humanos conforme dispositivos presentes na Resolução $n^{\circ}$ 466 de 12 de dezembro de 2012, do Conselho Nacional de Saúde/Comissão de Ética em Pesquisa/Ministério da Saúde, com a dispensa do Termo de Consentimento Livre e Esclarecido (TCLE), bem como o Termo de Autorização e Compromisso para uso de Informações, pois trata-se de um estudo que utilizou somente dados secundários obtidos a partir de um banco de dados.

\section{RESULTADOS}

Dos 1568 pacientes analisados, a neoplasia maligna do colo de útero (C53) foi a mais prevalente, com $19,57 \%$ dos diagnósticos. A segunda mais prevalente foi a neoplasia maligna de próstata (C61) com 16,96\%, seguida da neoplasia maligna da mama (C50) com $14,03 \%$ e neoplasia maligna do estômago (C16) com 12,43\%. As neoplasias malignas do cólon e reto e ânus e canal anal (C18-C21) foram agrupadas, representando 
$7,84 \%$ da prevalência. A neoplasia maligna da glândula tireoide (C73) apresentou 3,89\% de prevalência, seguida da neoplasia maligna da traqueia, brônquios e pulmões (C33-34), com 3,06\%; neoplasia maligna do ovário (C56) com $2,61 \%$ e neoplasia da cavidade oral (C00-C10) com 2,42\%. Por fim, os demais tipos de tumores sólidos foram agrupados na categoria OUTROS, com prevalência de $17,15 \%$. Com isso, estabeleceram-se os dez principais tumores sólidos mais prevalentes. A Tabela 1 evidencia as características gerais da amostragem com dados brutos sobre o sexo, faixa etária, raça/cor, cidade/procedência e situação do paciente e o Gráfico 1 demonstra a prevalência dos 10 Principais Tumores Sólidos no período de 2013 a 2017. (Tabela de Contingência $=10$ x 5; Qui-Quadrado=55.794; Graus de liberdade $=36(p)=0.0187)$. Entre os 10 principais tipos de câncer analisados ao longo de 5 anos os cânceres de colo de útero, próstata, mama, estômago e OUTROS apresentaram valores altamente significativos em relação aos demais (soma das categorias $=1568$ casos; $Q u i-Q u a d r a d o=651.834$; Grau de Liberdade=9; p-valor<0.0001).

Em relação ao ano de diagnóstico, 2017 apresentou o maior número com 390 casos $(24,87 \%)$, seguido de 2016 com 365 casos $(23,27 \%)$, mostrando efeitos significativos em relação aos tipos de cânceres, sendo que a Neoplasia do colo de útero, OUTROS e Neoplasia da próstata apresentaram os maiores valores. (Gráfico 2). (Tabela de Contingência=10 x 5; Qui-Quadrado=55.794; Graus de liberdade=36; $(p)=0.0187)$.

A tabela 2 mostra que, dos 1568 pacientes analisados, 877 são do sexo feminino (56\%) e 691, do masculino (44\%). Para o sexo feminino, as prevalências por cânceres foram colo de útero (35\%), mama $(24,85 \%)$, OUTROS $(13,79 \%)$, cólon e reto e ânus e canal anal $(7,18 \%)$, glândula tireoide $(6,27 \%)$, estômago $(5,58 \%)$, ovário $(4,67 \%)$, traqueia, brônquios e pulmões $(1,59 \%)$ e cavidade oral $(1,02 \%)$ e para o masculino foram os cânceres de próstata $(38,49 \%)$, OUTROS $(21,41 \%)$, estômago $(21,12 \%)$, cólon e reto e ânus e canal anal (8,68\%), traqueia, brônquios e pulmões $(4,92 \%)$, cavidade oral $(4,19 \%)$, glândula tireoide $(0,86 \%)$ e mama $(0,28 \%)$. A faixa etária foi altamente significativa em relação aos tipos de cânceres, sendo que para o sexo masculino, a faixa etária de 70 a 79 anos apresentou efeitos significativos para a Neoplasia Maligna da Próstata. Enquanto que para o sexo feminino, a faixa etária de 40 a 49 anos, apresentou efeito significativo para a Neoplasia Maligna do Colo do útero. (Tabela de Contingência=10 x 8; Qui-Quadrado de independência $=568.526$; Graus de liberdade =63; p-valor< 0.0001).

Em relação às cidades de procedência, Macapá (não especificada área urbana ou rural) foi a mais prevalente com 1053 do total $(67,15 \%)$, apresentando valores significativos às demais cidades, seguida por Santana com 219 (13,96\%) e Laranjal do Jari com 34 $(2,16 \%)$. As categorias Outros municípios do Amapá e Outros Estados apresentaram, respectivamente, $101(6,44 \%)$ e $14(0,89 \%)$. Entretanto, 147 dos diagnósticos $(9,37 \%)$ não foram informados os dados de procedência. (Gráfico 3) (Tabela de Contingência $=10 \times 6$, Qui-Quadrado=62.581; Graus de liberda$d e=45 ;(p)=0.0424)$.

A tabela 3 evidencia a relação dos tumores sólidos com a raça/cor, na qual 73,15\% 
Tabela 1 - Prevalência dos 10 Principais Tumores Sólidos em uma Unidade de Assistência de Alta Complexidade em Oncologia do Amapá (UNACON-AP) quanto ao Sexo, Faixa Etária, Raça/Cor, Cidade/Procedência e Situação do Paciente, no período de 2013 a 2017.

\begin{tabular}{|c|c|c|c|c|c|c|c|c|c|c|c|c|c|}
\hline \multirow[t]{3}{*}{ VARIÁVEIS } & \multicolumn{13}{|c|}{ DIAGNÓSTICO- CID-10 DAS NEOPLASIAS MALIGNAS } \\
\hline & \multirow[t]{2}{*}{\begin{tabular}{c|c}
$\begin{array}{c}\text { Colo } \\
\text { do } \\
\text { útero } \\
\text { (C53) }\end{array}$ & $\begin{array}{l}\mid \\
\text { n=307 }\end{array}$ \\
\multicolumn{2}{|c|}{}
\end{tabular}} & $\begin{array}{c}\text { Próstata } \\
\text { (C61) }\end{array}$ & \begin{tabular}{|l|} 
Mama \\
(C50)
\end{tabular} & \multicolumn{2}{|c|}{$\begin{array}{l}\text { Estômago } \\
\text { (C16) }\end{array}$} & \multirow[t]{2}{*}{$\begin{array}{l}\begin{array}{c}\text { Cólon e } \\
\text { reto e } \\
\text { ânus e } \\
\text { canal anal } \\
\text { (C18-C21) }\end{array} \\
n=123\end{array}$} & \multirow{2}{*}{$\begin{array}{c}\begin{array}{c}\text { Glândula } \\
\text { tireoide } \\
\text { (C73) }\end{array} \\
n=61\end{array}$} & \multirow[t]{2}{*}{$\begin{array}{c}\begin{array}{c}\text { Traqueia, } \\
\text { brônquios } \\
\text { e pulmões } \\
\text { (C33-C34) }\end{array} \\
n=48\end{array}$} & \multirow{2}{*}{$\begin{array}{l}\begin{array}{c}\text { Ovário } \\
\text { (C56) }\end{array} \\
n=41\end{array}$} & $\begin{array}{c}\text { Cavidade } \\
\text { oral (C00- } \\
\text { C10) }\end{array}$ & 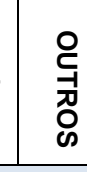 & \multicolumn{2}{|c|}{ TOTAL } \\
\hline & & $n=266$ & $\mathrm{n}=2$ & & $n=195$ & & & & & $n=38$ & $\mathrm{n}=269$ & $\mathrm{~F}$ & M \\
\hline \multicolumn{14}{|l|}{ SEXO } \\
\hline FEMININO & 307 & - & \multicolumn{2}{|c|}{218} & 49 & 63 & 55 & 14 & 41 & 9 & 121 & 877 & - \\
\hline MASCULINO & - & 266 & \multicolumn{2}{|c|}{2} & 146 & 60 & 6 & 34 & - & 29 & 148 & - & 691 \\
\hline \multicolumn{14}{|c|}{ FAIXA ETÁRIA (ANOS) } \\
\hline$<18$ & - & - & - & - & & 1 & - & - & - & - & 5 & 5 & 1 \\
\hline 18 a 29 & 17 & - & 5 & 7 & & 5 & 6 & 3 & 8 & 1 & 20 & 51 & 21 \\
\hline 30 a 39 & 67 & - & 36 & 11 & & 9 & 10 & 1 & 7 & 4 & 22 & 144 & 23 \\
\hline 40 a 49 & 94 & 3 & 70 & 28 & & 20 & 18 & 2 & 9 & 6 & 33 & 216 & 67 \\
\hline 50 a 59 & 65 & 18 & 58 & 52 & & 38 & 16 & 12 & 11 & 8 & 60 & 209 & 129 \\
\hline 60 a 69 & 38 & 84 & 29 & 51 & & 25 & 9 & 15 & 2 & 12 & 70 & 141 & 194 \\
\hline 70 a 79 & 23 & 116 & 16 & 34 & & 18 & 1 & 11 & 2 & 6 & 48 & 86 & 189 \\
\hline$\geq 80$ & 3 & 45 & 6 & 12 & & 7 & 1 & 4 & 2 & 1 & 11 & 25 & 67 \\
\hline \multicolumn{14}{|l|}{ RAÇA/COR } \\
\hline AMARELA & - & - & 1 & - & & - & - & - & 1 & - & 1 & 3 & - \\
\hline BRANCA & 5 & 5 & 4 & 2 & & 2 & - & 1 & 1 & - & 5 & 16 & 9 \\
\hline IGNORADO & 2 & 30 & 19 & 19 & & 8 & 1 & 4 & 6 & 3 & 19 & 50 & 61 \\
\hline $\begin{array}{c}\text { NÃO } \\
\text { PREENCHIDO }\end{array}$ & 254 & 175 & 139 & 138 & & 97 & 51 & 36 & 27 & 29 & 201 & 663 & 484 \\
\hline PARDA & 46 & 52 & 56 & 35 & & 15 & 8 & 7 & 5 & 6 & 40 & 141 & 129 \\
\hline PRETA & - & 4 & 1 & 1 & & 1 & 1 & - & 1 & - & 3 & 4 & 8 \\
\hline \multicolumn{14}{|c|}{ CIDADE/ PROCEDÊNCIA } \\
\hline $\begin{array}{l}\text { LARANJAL } \\
\text { DO JARI }\end{array}$ & 11 & 4 & 2 & 5 & & - & 1 & 1 & - & 1 & 9 & 21 & 13 \\
\hline MACAPÁ & 187 & 182 & 164 & 132 & & 93 & 39 & 33 & 27 & 25 & 171 & 591 & 462 \\
\hline $\begin{array}{c}\text { NÃO } \\
\text { INFORMADO }\end{array}$ & 43 & 24 & 11 & 10 & & 6 & 11 & 4 & 3 & 4 & 31 & 81 & 66 \\
\hline $\begin{array}{l}\text { OUTROS } \\
\text { ESTADOS }\end{array}$ & 5 & - & 5 & 1 & & 1 & - & - & 1 & - & 1 & 13 & 1 \\
\hline $\begin{array}{l}\text { OUTROS } \\
\text { MUNICÍPIOS } \\
\text { DO AP }\end{array}$ & 17 & 23 & 8 & 15 & & 8 & 4 & 2 & 4 & 2 & 18 & 46 & 55 \\
\hline SANTANA & 44 & 33 & 30 & 32 & & 15 & 6 & 8 & 6 & 6 & 39 & 125 & 94 \\
\hline \multicolumn{14}{|c|}{ SITUAÇÃO DO PACIENTE } \\
\hline $\begin{array}{c}\text { EM } \\
\text { SEGUIMENTO }\end{array}$ & 291 & 254 & 210 & 167 & & 117 & 59 & 32 & 38 & 34 & 240 & 813 & 629 \\
\hline $\begin{array}{c}\text { ÓBITO } \\
\text { ÓBITO/SEXO }\end{array}$ & 16 & 12 & 10 & 28 & & 6 & 2 & 16 & 3 & 4 & 29 & 64 & 62 \\
\hline $\mathrm{F}$ & 16 & - & 10 & 8 & & 3 & 2 & 7 & 3 & 1 & 14 & 64 & - \\
\hline M & - & 12 & - & 20 & & 3 & - & 9 & - & 3 & 15 & - & 62 \\
\hline
\end{tabular}

Fonte: Dados avaliados pelos autores e extraídos do SIGES-AP, 2018. 
Gráfico 1 - Prevalência dos 10 Principais Tumores Sólidos em uma Unidade de Assistência de Alta Complexidade em Oncologia do Amapá (UNACON-AP), no período de 2013 a 2017.

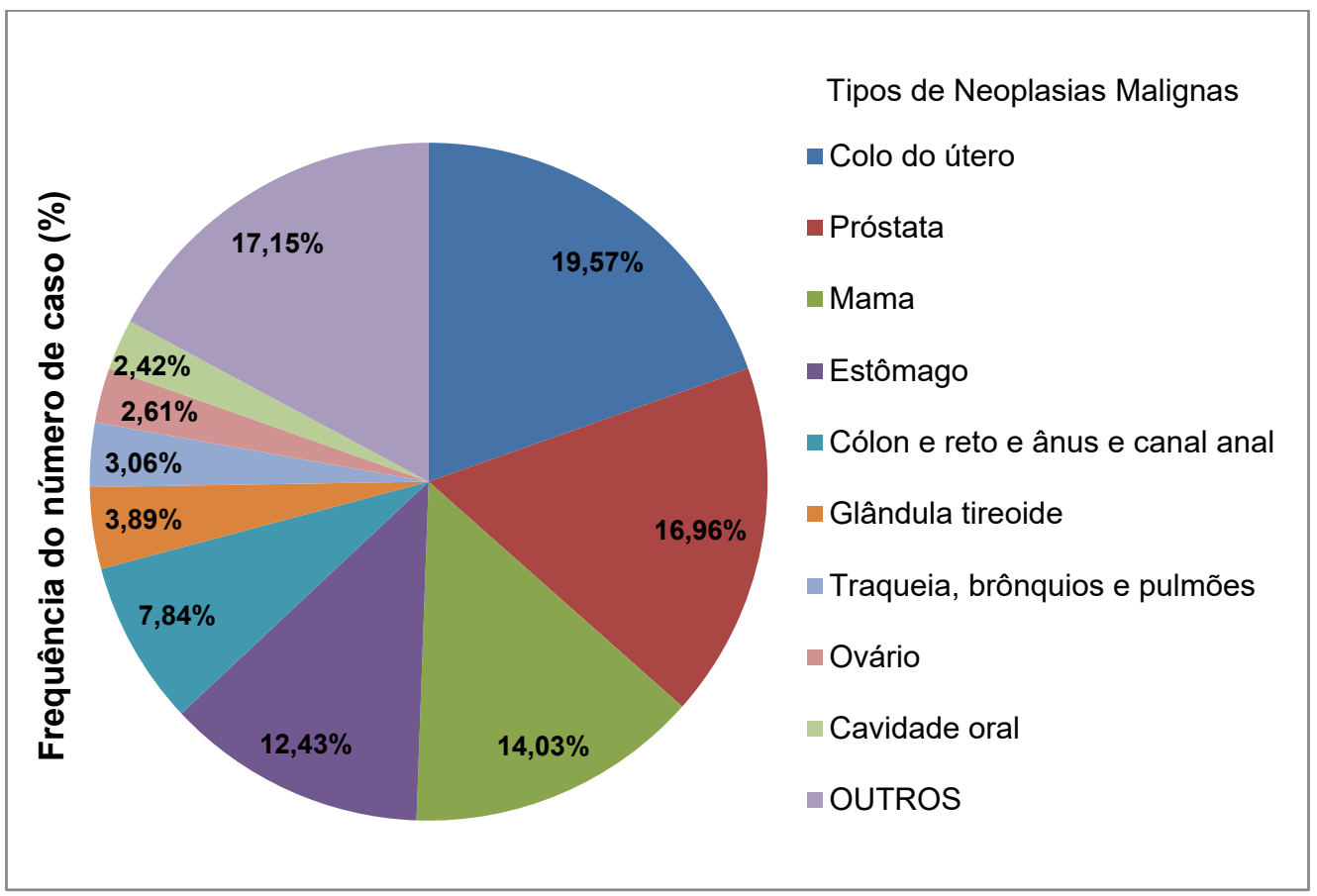

Fonte: Dados avaliados pelos autores e extraídos do SIGES-AP, 2018.

Gráfico 2 - Prevalência dos 10 Principais Tumores Sólidos em uma Unidade de Assistência de Alta Complexidade em Oncologia do Amapá (UNACON-AP) quanto ao Ano de Diagnóstico.

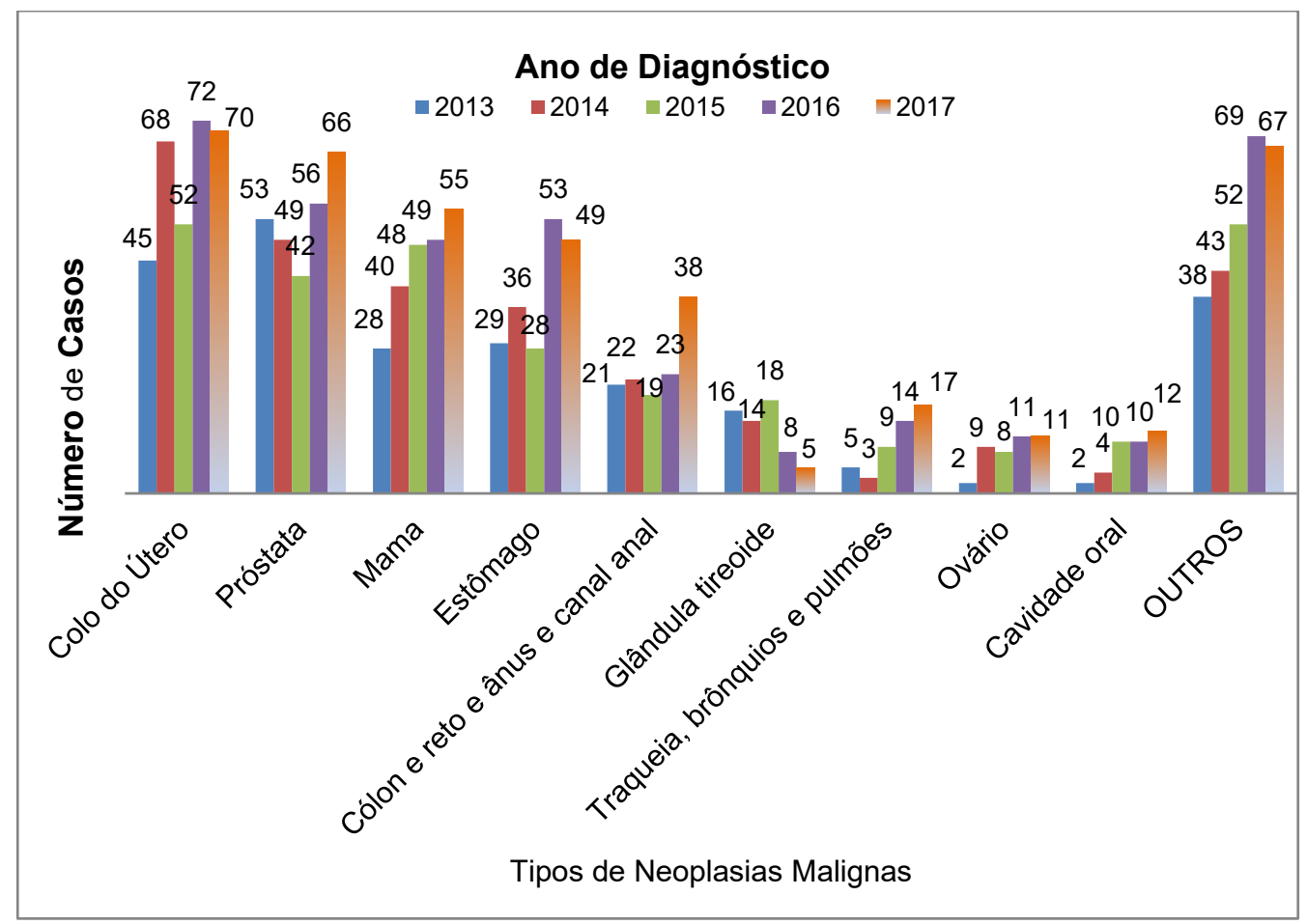

Fonte: Dados avaliados pelos autores e extraídos do SIGES-AP, 2018. 
Tabela 2 - Prevalência dos 10 Principais Tumores Sólidos em uma Unidade de Assistência de Alta Complexidade em Oncologia do Amapá (UNACON-AP), segundo a Faixa Etária, no período de 2013 a 2017.

\begin{tabular}{|c|c|c|c|c|c|c|c|c|}
\hline \multirow{2}{*}{$\begin{array}{c}\text { Tipos de Neoplasias Malignas no Sexo } \\
\text { Feminino }\end{array}$} & \multicolumn{8}{|c|}{ FAIXA ETÁRIA } \\
\hline & $<18$ & 18 a 29 & 30 a 39 & 40 a 49 & 50 a 59 & 60 a 69 & 70 a 79 & $\geq 80$ \\
\hline Colo do Útero $(n=307)$ & - & 17 & 67 & 94 & 65 & 38 & 23 & 3 \\
\hline Mama $(n=218)$ & - & 5 & 36 & 68 & 58 & 29 & 16 & 6 \\
\hline Estômago ( $n=49)$ & - & 5 & 7 & 6 & 7 & 12 & 9 & 3 \\
\hline Cólon e reto e ânus e canal anal $(n=63)$ & 1 & 1 & 4 & 7 & 21 & 17 & 9 & 3 \\
\hline Glândula tireoide (n-55) & - & 5 & 9 & 17 & 14 & 8 & 1 & 1 \\
\hline Traqueia, brônquios e pulmões ( $n=14)$ & - & 2 & 1 & - & 3 & 4 & 2 & 2 \\
\hline Ovário $(n=41)$ & - & 8 & 7 & 9 & 11 & 2 & 2 & 2 \\
\hline Cavidade oral $(n=9)$ & - & 1 & 1 & 3 & 1 & 2 & 1 & - \\
\hline OUTROS $(n=121)$ & 4 & 7 & 12 & 12 & 29 & 29 & 23 & 5 \\
\hline TOTAL (n=877) (56\%) & 5 & 51 & 144 & 216 & 209 & 141 & 86 & 25 \\
\hline Frequência do número de casos (\%) & 0,57 & 5,81 & 16,41 & 24,62 & 23,83 & 16,07 & 9,80 & 2,85 \\
\hline \multirow{2}{*}{$\begin{array}{l}\text { Tipos de Neoplasias Malignas no Sexo } \\
\text { Masculino }\end{array}$} & \multicolumn{8}{|c|}{ FAIXA ETÁRIA } \\
\hline & $<18$ & 18 a 29 & 30 a 39 & 40 a 49 & 50 a 59 & 60 a 69 & 70 a 79 & $\geq 80$ \\
\hline Próstata $(\mathrm{n}=266)$ & - & - & - & 3 & 18 & 84 & 116 & 45 \\
\hline Mama $(n=2)$ & - & - & - & 2 & - & - & - & - \\
\hline Estômago $(n=146)$ & - & 2 & 4 & 22 & 45 & 39 & 25 & 9 \\
\hline Cólon e reto e ânus e canal anal $(n=60)$ & - & 4 & 5 & 13 & 17 & 8 & 9 & 4 \\
\hline Glândula tireoide $(n=6)$ & - & 1 & 1 & 1 & 2 & 1 & - & - \\
\hline Traqueia, brônquios e pulmões ( $n=34$ ) & - & 1 & - & 2 & 9 & 11 & 9 & 2 \\
\hline Cavidade oral $(n=29)$ & - & - & 3 & 3 & 7 & 10 & 5 & 1 \\
\hline OUTROS $(n=148)$ & 1 & 13 & 10 & 21 & 31 & 41 & 25 & 6 \\
\hline TOTAL $(n=691)(44 \%)$ & 1 & 21 & 23 & 67 & 129 & 194 & 189 & 67 \\
\hline Frequência do número de casos (\%) & 0,14 & 3,03 & 3,32 & 9,69 & 18,66 & 28,07 & 27,35 & 9,69 \\
\hline
\end{tabular}

Fonte: Dados avaliados pelos autores e extraídos do SIGES-AP, 2018.

dos diagnósticos não tiveram tais informações preenchidas e $7,07 \%$ tiveram a informação ignorada. Dentre os restantes, prevaleceu a raça/cor parda com $17,21 \%$, seguida da branca com $1,59 \%$, preta com $0,76 \%$ e amarela com $0,19 \%$.

Referente à Situação do Paciente, $1442(91,96 \%)$ estavam em seguimento, sendo $56,38 \%$ mulheres e $43,62 \%$ homens e foram contabilizados 126 óbitos (8,03\%), com $50,79 \%$ dos casos do sexo feminino, contra $49,20 \%$ do masculino. Os casos de cânceres com pacientes em seguimento foram altamente significativos em relação aos casos de pacientes em óbito ao longo de 5 anos. (Gráfico 4) (Tabela de Contingência=10 x 2; 
Gráfico 3 -Prevalência dos 10 Principais Tumores Sólidos em uma Unidade de Assistência de Alta Complexidade em Oncologia do Amapá (UNACON-AP), segundo a Cidade/Procedência, no período de 2013 a 2017.

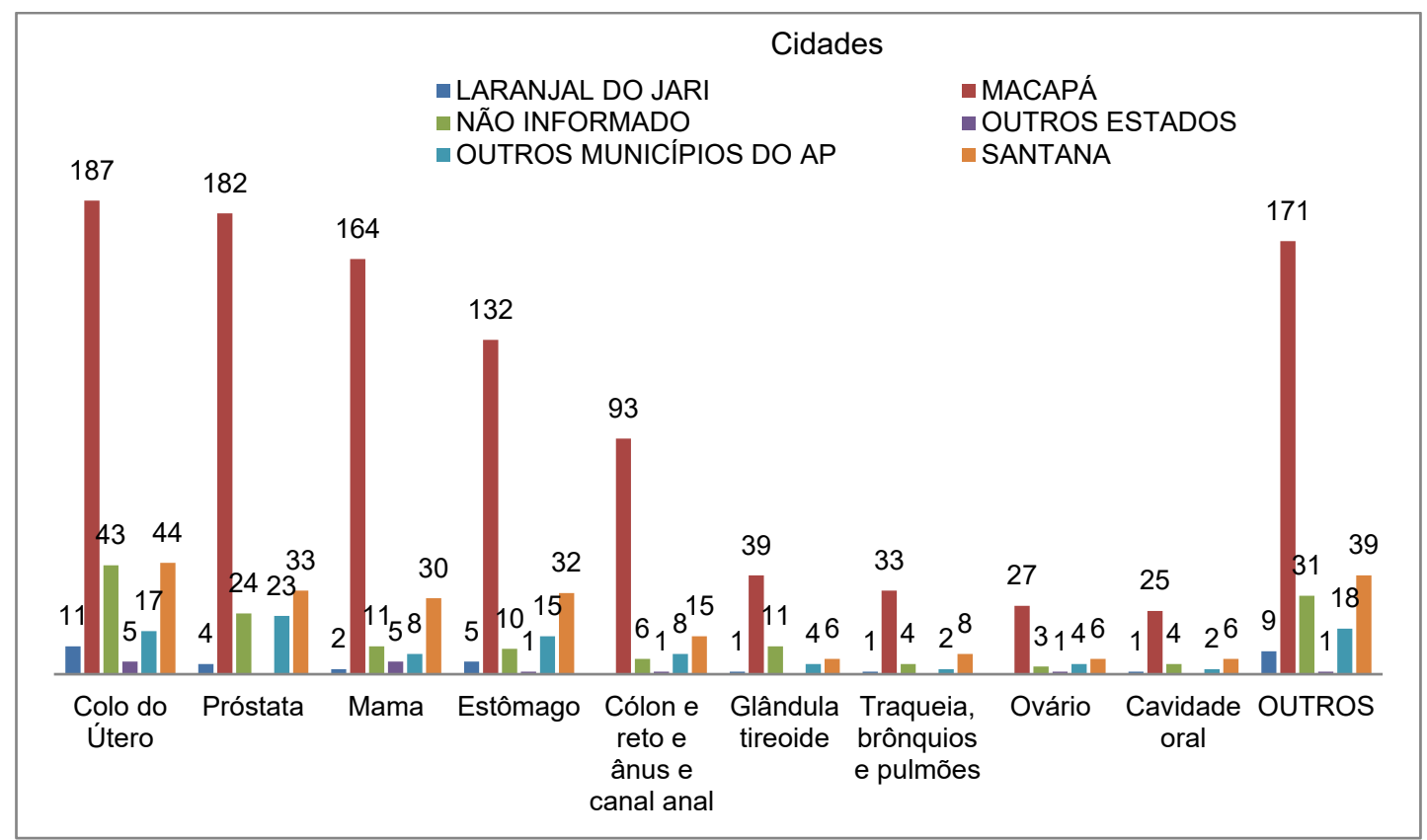

Fonte: Dados avaliados pelos autores e extraídos do SIGES-AP, 2018.

Tabela 3 - Prevalência dos 10 Principais Tumores Sólidos em uma Unidade de Assistência de Alta Complexidade em Oncologia do Amapá (UNACON-AP) quanto à Raça/Cor, no período de 2013 a 2017.

\begin{tabular}{|c|c|c|c|c|c|c|}
\hline \multirow[b]{2}{*}{ Tipos de Neoplasias Malignas } & \multicolumn{6}{|c|}{ RAÇA/COR } \\
\hline & AMARELA & BRANCA & IGNORADO & $\begin{array}{c}\text { NÃO } \\
\text { PREENCHIDO }\end{array}$ & PARDA & PRETA \\
\hline Colo do Útero $(n=307)$ & - & 5 & 2 & 254 & 46 & - \\
\hline Próstata $(n=266)$ & - & 5 & 30 & 175 & 52 & 4 \\
\hline $\operatorname{Mama}(n=220)$ & 1 & 4 & 19 & 139 & 56 & 1 \\
\hline Estômago (n=195) & - & 2 & 19 & 138 & 35 & 1 \\
\hline Cólon e reto e ânus e canal anal $(n=123)$ & - & 2 & 8 & 97 & 15 & 1 \\
\hline Glândula tireoide $(n=61)$ & - & - & 1 & 51 & 8 & 1 \\
\hline Traqueia, brônquios e pulmões ( $n=48$ ) & - & 1 & 4 & 36 & 7 & - \\
\hline Ovário $(n=41)$ & 1 & 1 & 6 & 27 & 5 & 1 \\
\hline Cavidade oral $(n=38)$ & - & - & 3 & 29 & 6 & - \\
\hline OUTROS $(n=269)$ & 1 & 5 & 19 & 201 & 40 & 3 \\
\hline TOTAL $(n=1568)$ & 3 & 25 & 111 & 1147 & 270 & 12 \\
\hline Frequência do número de casos (\%) & 0,19 & 1,59 & 7,07 & 73,15 & 17,21 & 0,76 \\
\hline
\end{tabular}

Fonte: Dados avaliados pelos autores e extraídos do SIGES-AP, 2018. 
Gráfico 4 - Prevalência dos 10 Principais Tumores Sólidos em uma Unidade de Assistência de Alta Complexidade em Oncologia do Amapá (UNACON-AP), quanto à Situação do Paciente, no período de 2013 a 2017.

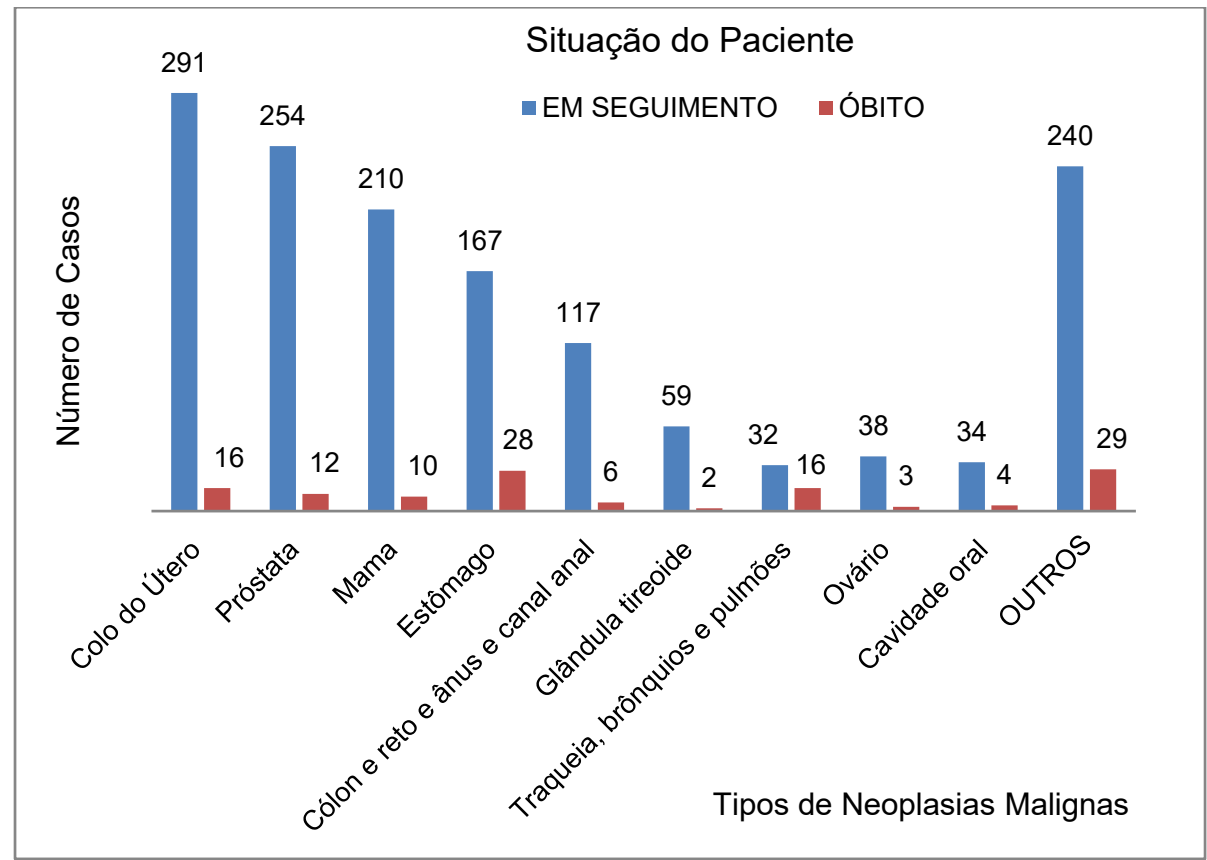

Fonte: Dados avaliados pelos autores e extraídos do SIGES-AP, 2018.

Qui-Quadrado=70.147; Graus de liberdade=9; p-valor<0.0001). E não houve valores significativos em relação aos casos de pacientes em óbito com o sexo ao longo de 5 anos. (Tabela de Contingência=7 x 2; Qui-Quadrado=9.976; Graus de liberdade $=6 ;(p)=0.1256)$.

\section{DISCUSSÃO}

Através dos dados coletados na UNACON-AP, analisaram-se 1568 pacientes com tumores sólidos, sendo $55,93 \%$ do sexo feminino e $44,86 \%$ do masculino, possuindo correspondência aproximada aos valores de $50,33 \%$ e $49,67 \%$, respectivamente, estimados para o ano de $2008^{18}$.

Em relação à faixa etária, a maior pre- valência foi entre 50 e 69 anos, intervalo dentro do qual se encontra a média de 51,9 anos descrita pelos dados da Pesquisa Nacional de Saúde (PNS) de 2013 ${ }^{19}$. Porém, houve certa discrepância quanto aos dados obtidos por Santos et al., 2017, segundo o qual o número de novos diagnósticos costuma prevalecer em maiores que 65 anos, enquanto, para a presente pesquisa, o quantitativo destacou-se para faixas etárias menores que a faixa dada como referência ${ }^{5}$.

Confirmando a transição demográfica, houve tendência de crescimento da incidência de novos casos para quase todos os anos, exceto 2016. A elevação está intimamente relacionada a áreas em desenvolvimento social e econômico e que apresentam crescimento 
populacional, como é o caso de Macapá e Santana, com os maiores quantitativos de diagnóstico, 1053 e 219 casos, respectivamente ${ }^{6,9,11,13}$.

Sobre a mortalidade, estima-se um aumento das taxas para as regiões Norte e Nordeste até o ano de 2030, enquanto, para as demais regiões, a mortalidade tenderá à estabilização ou, até mesmo, ao decréscimo ${ }^{12,13}$. Nesta pesquisa, houve um total de 126 óbitos (8,03\%), sendo $50,79 \%$ do sexo feminino e $49,20 \%$ do masculino, porém não houve significância na análise estatística entre a mortalidade e o sexo, uma vez que o número encontrado para esta análise foi pequeno e também o fato de que há cânceres que acometem somente um tipo de sexo ${ }^{6}$.

A PNS de 2013 também demonstrou que a prevalência de diagnósticos de câncer era maior entre pessoas que se autodeclaravam raça/cor branca, opondo-se à maioria de raça/cor parda $(17,21 \%)$ encontrada pela presente pesquisa. No entanto, é questionável a evidência dos resultados quanto a este parâmetro, diante da alta porcentagem $(73,15 \%)$ de pacientes que não tiveram esses dados preenchidos, evidenciando-se a dificuldade brasileira de manter e atualizar os registros sobre a doença em nossa população ${ }^{20}$.

O câncer do colo de útero apresentou o maior quantitativo com $19,57 \%$ dos diagnósticos e o mais prevalente para o sexo feminino (35\%). Apesar do câncer de mama ser o mais frequente, quando considerada a epidemiologia geral do Brasil, os dados tanto da prevalência quanto da mortalidade, confirmam a proporção mais elevada de câncer de colo de útero que é aguardada para os estados da região Norte, devido ao seu menor nível de desenvolvimento em relação às outras regiões do país $8,13,20,21$.

Por levar muitos anos para se desenvolver, o câncer de colo de útero é considerado raro em mulheres até 30 anos e sua incidência aumenta progressivamente até ter seu pico na faixa de 45 a 50 anos $^{22}$. Neste estudo, a faixa etária mais acometida foi de 40 a 49 anos $(30,61 \%)$, corroborando com os achados na literatura ${ }^{13}$. Portanto, é evidente a importância da ampliação de acesso, diagnóstico de qualidade e tratamento em tempo oportuno para esta região ${ }^{6}$.

Sobre os demais tipos de tumores sólidos, quando se excluiu OUTROS, a neoplasia maligna da próstata ocupou a segunda posição com $16,96 \%$ e a mais frequente para o sexo masculino $(38,49 \%)$, com faixa etária de 70 a 79 anos (43,60\%). Segundo Guerra et al. ${ }^{13}$, foi a primeira causa de óbito por sexo no Brasil e a segunda para o Amapá, corroborando com esta pesquisa para o segundo mais frequente no sexo masculino $(19,35 \%)$, porém ficou em terceiro lugar geral $(9,52 \%)$. Sua alta prevalência deve-se ao aumento na expectava de vida, melhorias nos recursos de diagnósticos e divulgação de informação sobre o tema, além de detecção precoce em homens assintomáticos ${ }^{13,23}$.

O câncer de mama ocupou a terceira posição geral $(14,03 \%)$, a segunda para o sexo feminino $(24,85 \%)$ e a última para o masculino $(0,28 \%)$. A faixa etária com maior prevalência foi entre 40 e 49 anos para ambos os sexos, enquanto, para a literatura, são aguardados valores maiores a partir dos 50 anos para mulheres e 60 anos para homens. A prevalência foi crescente durante os anos para o sexo feminino, refletindo 
melhoria no acesso à saúde e diagnóstico, enquanto para o sexo masculino permanece raro $^{24-26}$. Em pesquisa realizada por Guerra et al. ${ }^{13}$, o câncer de mama foi o mais frequente em mortalidade para o sexo feminino no Brasil, porém ocupando a quarta posição para o Amapá ${ }^{13}$. Os dados diferem com os achados neste estudo, uma vez que ocupou o quarto lugar geral $(7,93 \%)$ e o segundo mais frequente em mulheres $(15,62 \%)$, mas revelam uma taxa semelhante em estudo de Neves et al. ${ }^{8} \operatorname{com} 14,96 \%$.

O câncer de estômago é o segundo mais prevalente na região Norte, o quinto mais frequente nas mulheres e apresenta duas vezes mais frequência no sexo masculino ${ }^{14}$. No presente estudo, foi o quarto mais prevalente $(12,43 \%)$, ocupando a quinta posição para o sexo feminino $(5,58 \%)$ e o segundo para o masculino $(21,12 \%)$. As faixas etárias mais frequentes foram de 50 a 59 anos e 60 a 69 anos, para o sexo masculino e feminino, respectivamente. Sobre a mortalidade, a literatura aponta-o como o terceiro mais prevalente no sexo feminino e a primeira causa de óbito para o sexo masculino no Amapá, reforçando os dados encontrados nesta pesquisa, com $12,50 \%$ para o feminino e $32,25 \%$ para o masculino ${ }^{13}$. Sua alta incidência está relacionada aos maus hábitos alimentares e baixo saneamento básico e possui risco de desenvolvimento maior após os 50 anos $^{27}$.

O câncer colorretal ocupou a quinta posição geral com 7,84\% e o terceiro lugar e com faixa etária mais acometida entre 50 e 59 anos para ambos os sexos. A mortalidade ficou em quinto lugar para o sexo feminino e em quarto para o masculino. Os resultados encontrados correspondem aos encontrados na literatura, com prevalência crescente que corresponde ao aumento da cobertura do rastreamento de câncer colorretal por colonoscopia, preconizado a partir dos 50 $\operatorname{anos}^{17,28,29}$.

\section{CONCLUSÃO}

Através do presente estudo, pode-se concluir que o perfil epidemiológico dos indivíduos com câncer no estado é caracterizado por maioria do sexo feminino, parda, na faixa etária mais prevalente para ambos os sexos de 50 a 59 anos, de maioria proveniente da capital.

Além disso, evidenciou-se a influência sociodemográfica na epidemiologia do câncer. Enquanto a maioria das regiões brasileiras tende a se aproximar dos padrões aguardados para países de alta renda, a região Norte, em especial o Amapá, ainda insere-se nos padrões populacionais de baixa renda, fato este evidenciado quando se tem o câncer de colo de útero como o mais prevalente e idades entre 40 a 49 anos.

Por fugir à regra nacional em alguns aspectos, a região Norte, em especial o Estado do Amapá, merece um maior incentivo à pesquisa do câncer, no que se refere a uma melhor abordagem no registro de dados, tanto no prontuário, quanto no sistema de registro SIGES, com capacitação da equipe responsável pelo registro de tais informações, pois estas tornam-se fatores limitadores quando o objetivo é coleta de dados epidemiológicos. A implementação de dados relevantes no registro informatizado da UNACON-AP e no próprio prontuário, como: Estado civil, religião, 
escolaridade, profissão, local de trabalho, dados clínicos (tabagista, etilista, comorbidades, história de câncer na família, localização do tumor primário, tipo histológico, estadiamento, tratamento, seguimento do paciente), são extremamente importantes, uma vez que são determinantes para se compreender o processo saúde/doença e a epidemiologia de uma população.

\section{REFERÊNCIAS}

1. Lobato LL, Cabral SS, Vanzeler TL, Nascimento RE, Picanço OM, Rodrigues ASN. Identification of Thr241Met Polymorphisms of the XRCC3 Gene in People with Gastric Cancer in the Amazon Region, Brazil. Mol Genet Med. 2017; 11(3): 1-3.

2. Cabral SS, Lobato LL, Nascimento RE, Picanço OM, Rodrigues ASN. Association between XRCC3 Thr241Met Polymorphism and the Risk of Cancer in Northern Brazil. BJMMR. 2016; 15(7): 1-6.

3. Domingos PAS, Passalacqua MLC, Oliveira ALBM. M. Câncer bucal: um problema de saúde pública. Rev Odontol Univ Cid São Paulo. 2014; 26(1): 46-52.

4. Wang J-Y, Liang Y-W, Yeh CC, Liu CS, Wang C-Y. Time dependent risks of cancer clustering among couples: a nationwide population based cohort study in Taiwan. BMJ Open. 2018; 8(e018968): 1-10.

5. Santos EGA, Souza JC, Santos ALS, Santos MIPO, Oliveira TNC. Perfil clínico-epidemiológico de idosos submetidos à quimioterapia antineoplásica atendidos em um hospital de referência oncológica do estado do Pará, Brasil. Rev Pan-Amaz Saude. 2017; 8(2): 47-56.

6. Barbosa IR, Costa ICC, Perez MMB, Souza DLB. Desigualdades socioespaciais na distribuição da mortalidade por câncer no Brasil. Hygeia. 2016; 12(23): 122-132.

7. Guerra MR, Gallo CVM, Mendonça GAS. Risco de câncer no Brasil: tendências e estudos epidemiológicos mais recentes. Revista Brasileira de Cancerologia. 2005; 51(3): 227-234.

8. Neves RR, Fernandes NM, Martins FR, Junior CAR, Dias FCR, Cuellar PMG. Panorama dos casos de câncer atendidos no hospital geral público de Palmas, Tocantins, Brasil. Revista de Patologia do Tocantins. 2017; 4(3): 22-26.

9. Aguiar JR PN, Stock GT, Lopes JR. GL, Almeida MS, Tadokoro H, Gutierres BS, et al. Disparidades na epidemiologia e no tratamento de câncer nas populações indígenas brasileiras. Einstein. 2016; 14(3): 330-337.
Diante do exposto, fica clara a importância da melhoria nos registros hospitalares sobre o câncer e da melhor execução das políticas públicas destinadas a essa doença, em vista não apenas do diagnóstico e tratamento adequados, mas da prevenção, promoção integral de saúde e de dignidade de toda a população que compõe a sociedade amapaense.

10. Mcmullin J. Cancer. Annu. Rev. Anthropol. 2016 45:251-66.

11. Machado BM, Andrade IHR, Martins PT, Clarindo PMN, Matos MR. Casos oncológicos no município de Valença: um estudo epidemiológico. Saber Digital. 2017; 10(2), 27-42.

12. Barros MC, Pinheiro ITR, Oliveira LA, Santos WRCC Carvalho CA. Evolução da mortalidade por câncer de fígado e colorretal segundo sexo e regiões do Brasil de 1990 a 2014. Revista Ceuma Perspectivas. 2017; 29(1):88-97.

13. Guerra MR, Bustamante-Teixeira MT, Corrêa CSL, Abreu DMX, Curado MP, Mooney M, et al. Magnitude e variação da carga da mortalidade por câncer no Brasil e Unidades da Federação, 1990 e 2015. Rev Bras Epidemiol 2017; 20(SUPPL 1):102-115.

14. Instituto Nacional de Câncer José Alencar Gomes da Silva - INCA. Estimativa 2018: incidência de câncer no Brasil. Disponível em: http://www.inca.gov.br/ estimativa/2018/estimativa-2018.pdf. Acesso em: 22 jullho de 2018.

15. Bray F, Ren J-S, Masuyer E, Ferlay J. Global estimates of cancer prevalence for 27 sites in the adult population in 2008. Int. J. Cancer. 2013; 132:1133-1145.

16. Maddams J, Utley M, Moller H. Projections of cancer prevalence in the United Kingdom, 2010-2040. British Journal of Cancer. 2012; 107:1195-1202.

17. Barros MBA, Francisco PMSB, Zanchetta LM, César CLG. Tendências das desigualdades sociais e demográficas na prevalência de doenças crônicas no Brasil, PNAD: 2003- 2008. Ciência \& Saúde Coletiva. 2011; 16(9):3755-3768.

18. Mergen CTT, Strassburger SZ. Prevalência dos tipos de câncer em pacientes atendidos em um Centro de Alta Complexidade em Oncologia. Revista Contexto \& Saúde. 2009; 9(17):31-38. 
19. Silva MJS, Lima LFT, O'Dwyer G, Osorio-de-Castro CGS. Política de Atenção ao Câncer no Brasil após a Criação do Sistema Único de Saúde. Revista Brasileira de Cancerologia. 2017; 63(3):177-187.

20. Oliveira MM, Malta DC, Guauche H, Moura L, Silva GA. Estimativa de pessoas com diagnóstico de câncer no Brasil: dados da Pesquisa Nacional de Saúde, 2013. Rev Bras Epidemiol. 2015; 18(SUPPL 2):146-157.

21. Girianelli VR, Gamarra CJ, Silva GA. Os grandes contrastes na mortalidade por câncer do colo uterino e de mama no Brasil. Rev Saúde Pública. 2014; 48(3):459-467.

22. Silva DSM, Silva AMN, Brito LMO, Gomes SRL, Nascimento MDSB, Chein MBC. Rastreamento do câncer do colo do útero no Estado do Maranhão, Brasil. Ciência \& Saúde Coletiva. 2014, 19(4):1163-1170.

23. Barbosa IR. Tendências e projeções da mortalidade pelos cânceres específicos ao gênero no Brasil [dissertação]. Natal (RN): Universidade Federal do Rio Grande do Norte; 2015.

24. Barbosa AMM, Ferraz EB, Hott GO, Gomes JGE, Paulabonfá L, Oliveira SR et al. Câncer de mama, um levantamento epidemiológico dos anos de 2008 a 2013. Revista Científica do ITPAC. 2017; 10(2):52-61.

25. Girianelli VR, Gamarra CJ, Silva GA. Os grandes contrastes na mortalidade por câncer do colo uterino e de mama no Brasil. Rev Saúde Pública. 2014; 48(3):459-467.
26. Bonfim RJA, Vidal FCB, Silva DF, Silva MACN, Mubárack TC, Bezerra GFB et al. Câncer de mama no homem: análise dos aspectos epidemiológicos, clínicos e terapêuticos em serviço formal brasileiro. Revista Brasileira de Oncologia Clínica. 2014; 10(37):90-96.

27. Guimaraes AVS, Sales ML. Fatores de risco no desenvolvimento de câncer gástrico: uma análise na população brasileira. Revista Brasileira de Ciências da Vida. 2017; 5(1):1-24.

28. Helena FGS, Carvalho LP, Guimarães MRF, Miranda B. Atuais diretrizes do rastreamento do câncer colorretal: revisão de literatura. Revista da AMRIGS. 2017; 61 (1):76-83.

29. Pelizzer T, Dias CP, Poeta J, Torriani T, Roncada C. Prevalência de câncer colorretal associado ao papilomavírus humano: uma revisão sistemática com metanálise. Rev Bras Epidemiol. 2016; 19(4):791-802.

\section{CORRESPONDÊNCIA}

Dielle de Carvalho Loureiro

Avenida Presidente Marechal Humberto de Alencar Castelo Branco, 1561.

Central. Santana. Amapá-AP

CEP: 68.925-225

E-mail: dielle_loureiro@hotmail.com 